\title{
Fratura na coluna vertebral por mieloma múltiplo: correlação entre sobrevida e índices de Tomita e Tokuhashi
}

Multiple myeloma and vertebral fractures: correlation between survival rate and Tomita and Tokuhashi scores Fractura de la columna vertebral por mieloma múltiple: correlación entre sobrevida e índices de Tomita y Tokuhashi

\author{
Osmar Avanzi' \\ Elcio Landim ${ }^{2}$ \\ Robert Meves ${ }^{3}$ \\ Maria Fernanda Silber Caffaro 4 \\ Marcos Vaz de Lima ${ }^{5}$
}

\section{RESUMO}

Objetivo: o mieloma múltiplo (MM) é a neoplasia óssea primária mais freqüente na coluna vertebral. Em razão da grande morbi-mortalidade destas lesões, discute-se qual o melhor tratamento nestes pacientes. Na prática, observamos similaridade das opções de tratamento entre os pacientes portadores de metástases ósseas e MM. Os índices de sobrevida de Tomita e Tokuhashi são utilizados com o intuito de auxiliar na escolha do tratamento nos portadores de metástases. Faltam estudos sobre a aplicabilidade destes índices em pacientes portadores de MM. Neste trabalho vamos avaliar a aplicabilidade dos índices de Tomita e Tokuhashi nos pacientes portadores

\begin{abstract}
Objective: multiple myeloma (MM) is the most common primary neoplasm tumor in the spine. Due to the high morbidity and mortality of these spinal lesions, it is of important to discuss the best treatment in these patients. In clinical practice, there are similarities in the treatment options for patients with bone metastasis and $M M$. The survival rates of Tomita and Tokuhashi scores are used in order to assist in the choice of treatment patients with metastasis. No studies about the applicability of these scores in patients with MM were found. The objective of this study is to evaluate the applicability of Tomita and Tokuhashi scores in patients with
\end{abstract}

\section{RESUMEN}

Objetivo: el Mieloma Múltiple (MM) es la neoplasia ósea primaria más frecuente en la columna vertebral. En razón de la gran morbimortalidad de estas lesiones, se discute cuál sería el mejor tratamiento en estos pacientes. En la práctica observamos semejanza de las opciones de tratamiento entre los pacientes portadores de metástasis óseas y MM. Los indices de sobrevida de Tomita y Tokuhashi son utilizados con el objetivo de auxiliar en la opción del tratamiento en los portadores de metástasis. Faltan estudios sobre la aplicabilidad de estos índices en pacientes portadores de MM. En este trabajo vamos a evaluar la aplicabilidad de

\footnotetext{
Trabalho realizado no Departamento de Ortopedia e Traumatologia da Faculdade de Ciências Médicas da Santa Casa de Misericórdia de São Paulo - FCMSCMSP - São Paulo (SP), Brasil.

'Consultor do Grupo de Coluna e Diretor do Departamento de Ortopedia e Traumatologia da Faculdade de Ciências Médicas da Santa Casa de São Paulo FCMSCSP - São Paulo (SP), Brasil.

${ }^{2}$ Chefe do Grupo de Coluna do Departamento de Ortopedia e Traumatologia da Faculdade de Ciências Médicas da Santa Casa de São Paulo - FCMSCSP - São Paulo (SP), Brasil.

${ }^{3}$ Assistente do Grupo de Coluna do Departamento de Ortopedia e Traumatologia da Faculdade de Ciências Médicas da Santa Casa de São Paulo - FCMSCSP São Paulo (SP), Brasil.

${ }^{4}$ Professora Instrutora e Assistente do Grupo de Coluna do Departamento de Ortopedia e Traumatologia da Faculdade de Ciências Médicas da Santa Casa de São Paulo FCMSCSP - São Paulo (SP), Brasil.

${ }^{5}$ Médico Estagiário do Grupo de Coluna do Departamento de Ortopedia e Traumatologia da Faculdade de Ciências Médicas da Santa Casa de São Paulo FCMSCMSP - São Paulo (SP), Brasil.
} 
de MM e lesão vertebral. Métodos: estudo retrospectivo mediante avaliação de prontuários e radiografias de portadores de MM por meio da aplicação dos critérios de Tomita e Tokuhashi. Resultados: em um ano, $19(63,3 \%)$ estavam vivos, em dois anos $13(43,3 \%)$ e em cinco anos quatro $(13,3 \%)$ pacientes estavam vivos. Não houve correlação entre os índices (Tomita e Tokuhashi) e a taxa de sobrevida nestes pacientes $(p=0,2255)$. Conclusão: há necessidade de adaptação dos índices de Tomita e Tokuhashi para apresentarem aplicabilidade nos portadores de MM na coluna.
MM and vertebral lesions. Methods: Retrospective study on evaluation of radiographs and medical records of patients with MM using the criteria of Tomita and Tokuhashi. Results: At 1 year 19 (63.3\%) were alive, 2 years 13 (43.3\%) and 5 years $4(13.3 \%)$ patients were alive. There was no correlation between the scores (Tomita and Tokuhashi) and survival rate in these patients ( $p=0.2255)$. Conclusion: There is a need for adjustment or alterations of the scores of Tomita and Tokuhashi to have applicability in patients with MM in column.

los índices de Tomita y Tokuhashi en los pacientes portadores de MM y lesión vertebral. Métodos: estudio retrospectivo mediante evaluación de historias clínicas y radiografías de portadores de MM por medio de la aplicación de los criterios de Tomita y Tokuhashi. Resultados: En un año 19 estuvieron vivos (63.3\%), en 2 años fueron 13 (43.3\%) y en 5 años fueron 4 los que estuvieron vivos (13.3\%). No hubo correlación entre los indices (Tomita y Tokuhashi) y la tasa de sobrevida en estos pacientes $(p=0.2255)$. Conclusión: Hay necesidad de adaptación de los indices de Tomita y Tokuhashi para presentar una aplicabilidad en los portadores de MM en la columna.

KEYWORDS: Prognosis; Neoplasm metastasis; Spinal neoplasms/ secondary; Multiple myeloma; Life expectancy; Outcome assessment
DESCRIPTORES: Pronóstico; Metástasis de la neoplasia; Neoplasias de la columna vertebral/secundario; Mieloma múltiple; Esperanza de vida; Evaluación de resultado de resultados

\section{INTRODUÇÃO}

O Mieloma Múltiplo (MM) é a neoplasia óssea primária representada pela proliferação maligna dos plasmócitos na medula óssea ${ }^{1-4}$. Sua incidência é 5-10/100.000 pessoas por ano. A lesão solitária ou plasmocitoma é diagnosticada em apenas $10 \%$ destes pacientes ${ }^{3,5}$. As complicações descritas mais comuns do MM são hipercalcemia, dor óssea e fraturas em ossos do esqueleto apendicular e axial. O comprometimento vertebral nas metástases e no MM prejudicam não só a função mecânica de sustentação da coluna, mas também a qualidade de vida destes pacientes, em decorrência da presença de quadro doloroso ou da lesão neurológica associada ${ }^{1}$ $\left.{ }^{3,5-10}\right)$. No intuito de padronizar a conduta e tentar estabelecer o prognóstico nos portadores de metástase na coluna, dois sistemas de avaliação foram desenvolvidos: o de Tomita et al. e o de Tokuhashi et al. ${ }^{2}$, 7, 11, 12. A correlação entre a taxa de sobrevida e estes índices é contestada por muitos autores, e sua aplicabilidade para o MM ainda não foi avaliada ${ }^{6,13-16}$. Em virtude da semelhança de comportamento, história natural e de tratamento entre os portadores de metástase e MM na coluna, estudamos a aplicabilidade destes índices nos portadores de fratura patológica por MM na coluna. Em tese, como nas metástases, quanto maior o comprometimento do estado geral e disseminação da doença (variáveis analisadas nestes índices) pior a sobrevida nos portadores de MM. A escolha do tipo de tratamento nestes casos continua um desafio, mas pode ser facilitada com a padronização da conduta baseada em escores como o de Tomita e o de Tokuhashi.
O objetivo deste trabalho é determinar a taxa de sobrevida e sua possível correlação com os índices de gravidade de Tomita e Tokuhashi nos pacientes portadores de MM com lesão vertebral.

\section{MÉTODOS}

Foram avaliados, retrospectivamente, após aprovação do Comitê de Ética da Faculdade de Ciências Médicas da Santa Casa de São Paulo (FCMSCSP), 30 pacientes portadores de mieloma múltiplo diagnosticados por meio de mielograma. Foram incluídos neste estudo pacientes portadores de fratura patológica por MM na coluna identificada por radiografias, tomografia axial computadorizada ou ressonância magnética. Foram excluídos os pacientes com prontuários incompletos.

O escore de Tokuhashi ${ }^{7}$ foi desenvolvido para indicar o tipo de tratamento em lesões metastáticas da coluna vertebral, levando em conta os seguintes critérios: 1. Condição geral do paciente, segundo Karnofsky ${ }^{10}$ : pobre, 0 ponto; moderada 1 ponto e boa, 2 pontos; 2 . Número de metástases ósseas extra-espinhais: maior ou igual a três, 0 ponto; uma ou duas, 1 ponto e nenhuma, 2 pontos; 3 . Número de metástases vertebrais: maior ou igual a três, 0 pontos; duas, 1 ponto e uma, 2 pontos; 4 . Ressecabilidade de metástases em órgãos nobres: irressecável, 0 ponto; ressecável, 1 ponto e ausente, 2 pontos; 5 . Sítio primário do tumor: pulmão e estômago, 0 pontos; rim, fígado 
e útero, 1 ponto e tireóide, próstata, mama, reto, outros e não-identificados, 2 pontos; e 6 . Comprometimento neurológico medular: completo, 0 ponto, incompleto, 1 ponto e ausente, 2 pontos. Para cada um dos critérios é dada uma nota de 0 a 2, totalizando com os seis parâmetros, portanto, 12 pontos (Tabela 1). Posteriormente, o autor modificou o escore, alterando o parâmetro sítio primário do tumor, que agora varia de 0 a 5 pontos, em virtude da relevância do mesmo no prognóstico destes pacientes ${ }^{2,11}$.

Após esta modificação a nova pontuação ficou da seguinte forma: pulmão, osteossarcoma (que apesar de tumor primário ósseo foi incluído na nova lista), estômago, bexiga, esôfago e pâncreas, 0 pontos; fígado, ureter e não identificados, 1 ponto; outros, 2 pontos; rim e útero, 3 pontos; reto, 4 pontos; e tireóide, mama, próstata e carcinóide, 5 pontos. Tokuhashi et al. com base nestes índices indicam, respectivamente, o prognóstico e a opção de tratamento nestes pacientes: a) 0 a 8 pontos, prognóstico de até 6 meses, tratamento conservador ou paliativo em casos isolados; b) 9 a 11 pontos, prognóstico maior de 6 meses, tratamento paliativo ou cirurgia excisional em casos de lesão única e sem metástases para órgãos nobres; c) 12 a 15 pontos, prognóstico maior de 1 ano, tratamento com cirurgia excisional (Tabela 2).

O escore de Tomita ${ }^{17}$ usa uma pontuação segundo três fatores prognósticos: 1 . Grau de malignidade do tumor primário de acordo com o crescimento (lento, 1 ponto; moderado,

\section{TABELA 1 - O Índice de Tokuhashi et al.}

\begin{tabular}{|c|c|}
\hline & Nota \\
\hline \multicolumn{2}{|l|}{ Condição geral } \\
\hline Pobre & 0 \\
\hline Moderada & 1 \\
\hline Boa & 2 \\
\hline \multicolumn{2}{|l|}{ Metástases ósseas extra-espinais } \\
\hline$\geq 3$ & 0 \\
\hline $1-2$ & 1 \\
\hline 0 & 2 \\
\hline \multicolumn{2}{|l|}{ Metástases vertebrais } \\
\hline$\geq 3$ & 0 \\
\hline 2 & 1 \\
\hline 1 & 2 \\
\hline \multicolumn{2}{|l|}{ Metástases de órgãos nobres } \\
\hline Irressecáveis & 0 \\
\hline Ressecáveis & 1 \\
\hline Ausentes & 2 \\
\hline \multicolumn{2}{|l|}{ Sítio primário } \\
\hline Pulmão e estômago & 0 \\
\hline Rim, fígado, útero, outros, não-identificados & 1 \\
\hline Tireóide, próstata, mama, reto & 2 \\
\hline \multicolumn{2}{|l|}{ Lesão neurológica } \\
\hline Completa & 0 \\
\hline Incompleta & 1 \\
\hline Ausente & 2 \\
\hline
\end{tabular}

2 pontos e rápido, 4 pontos); 2. Presença de metástase visceral (sem metástase, 0 ponto; tratável, 2 pontos; intratável, 4 pontos) e 3. Presença de metástase óssea (solitária ou isolada, 1 ponto; múltipla, 2 pontos). A soma total pode variar entre 2 e 10 pontos (Tabela 3). De acordo com Tomita et al.12 a estratégia de tratamento será: a) 2 a 3 pontos, excisão ampla ou marginal para controle local a longo prazo; b) 4 a 5 pontos, excisão marginal ou intralesional para controle local a médio prazo; c) 6 a 7 pontos, cirurgia paliativa para controle a curto prazo; d) 8 a 10 pontos, tratamento não-cirúrgico (Tabela 4).

Como o MM não foi incluído nos estudos originais de Tomita et al e Tokuhashi et al. realizamos algumas adaptações para tornar possível a inclusão da doença neste estudo. Quanto à pontuação do foco primário, procurou-se nos escores originais o foco primário com sobrevida semelhante ao MM, que é de seis meses nos casos em que há comprometimento vertebral. Trata-se do câncer do fígado, que recebe a nota um em ambos os escores. Portanto, o MM recebeu a mesma nota. Quanto ao acometimento visceral e esquelético apendicular, consideramos a hepatomegalia (que atinge até $20 \%$ dos pacientes) e fraturas patológicas, respectivamente. A identificação das mesmas foi feita através de radiografias simples do corpo inteiro.

Analisamos os índices prognósticos, a sobrevida esperada e a sobrevida real por meio das curvas de sobrevida e do método de Kaplan-Meyer. A sobrevida foi verificada no dia 15/5/2007 nos arquivos do SAME-FCMSCSP.

TABELA 2
$\begin{aligned} & \text { TAcordo } \\
& \text { acoramento e prognóstico de }\end{aligned}$
\begin{tabular}{lll}
\hline Resultado & Prognice de Tokuhtico & Tratamento \\
\hline 0 a 8 pontos & 6 meses & Conservador \\
9 a 11 pontos & $6-12$ meses & Paliativo ou excisional \\
12 a 15 pontos & mais de 12 meses & Excisional \\
\hline
\end{tabular}

TABELA 3 - O índice de Tomita et al.

\begin{tabular}{lc}
\hline & Nota \\
\hline Grau de malignidade & 1 \\
Lento & 2 \\
Moderado & 4 \\
Rápido & \\
Metástase visceral & \\
Ausente & 0 \\
Tratável & 2 \\
Intratável & 4 \\
Metástase óssea & \\
Solitária & \\
Múltipla & 1 \\
& 2
\end{tabular}


TABELA 4 - Estratégia de tratamento de acordo com o índice de Tomita et al.

\begin{tabular}{ll}
\hline Resultado & Estratégia de tratamento \\
\hline 2-3 pontos & $\begin{array}{l}\text { Excisão ampla ou marginal, controle a } \\
\text { longo prazo }\end{array}$ \\
4-5 pontos & $\begin{array}{l}\text { Excisão marginal ou intra-lesional, } \\
\text { controle a médio prazo }\end{array}$ \\
6-7 pontos & $\begin{array}{l}\text { Cirurgia paliativa, controle a curto } \\
\text { prazo }\end{array}$ \\
8-10 pontos & Tratamento não-cirúrgico \\
\hline
\end{tabular}

\section{RESULTADOS}

As notas dos parâmetros de todos os pacientes estão ilustradas na Tabela 5. Foram calculados os escores de Tomita e Tokuhashi para os pacientes (Tabelas 2 e 3). Em um ano, 19 pacientes $(63,3 \%)$ estavam vivos, em dois anos $13(43,3 \%)$ e em cinco anos $4(13,3 \%)$ pacientes estavam vivos.

Índice deTokuhashi - Para a variável condição geral, dois pacientes $(6,7 \%)$ receberam nota 0 (condição geral pobre), nove pacientes $(30 \%)$ receberam nota 1 (condição moderada) e $19(63,3 \%)$ receberam nota 2 (condição boa). Para a variável metástases ósseas, dez pacientes $(33,3 \%)$ apresentavam três ou mais focos (nota 0$)$, dois $(6,7 \%)$ apresentavam um ou dois focos (nota 1) e 18 (60\%) não apresentaram outras lesões (nota 2). Quanto às metástases vertebrais, 17 pacientes $(56,6 \%)$ tinham acometimento de três

TABELA 5 - Notas de cada parâmetro dos escores de todos os pacientes $(n=30)$

\begin{tabular}{|c|c|c|c|c|c|c|c|c|c|c|c|c|}
\hline \multicolumn{8}{|c|}{ Tokuhashi } & \multicolumn{5}{|c|}{ Tomita } \\
\hline Paciente $\mathrm{n}^{\circ}$ & CG & MO & MV & OV & $1^{\circ}$ & $\mathrm{N}$ & TOT & $1^{\circ}$ & OV & MO & TOT & Sobrevida atual meses \\
\hline 1 & 1 & 2 & 0 & 2 & 1 & 1 & 7 & 1 & 0 & 2 & 3 & 120 \\
\hline 2 & 2 & 0 & 1 & 2 & 1 & 2 & 7 & 1 & 0 & 2 & 3 & 12 \\
\hline 3 & 2 & 0 & 0 & 2 & 1 & 1 & 6 & 1 & 0 & 2 & 3 & 84 \\
\hline 4 & 2 & 0 & 2 & 2 & 1 & 1 & 8 & 1 & 0 & 1 & 2 & 48, óbito \\
\hline 5 & 1 & 2 & 0 & 2 & 1 & 1 & 7 & 1 & 0 & 2 & 3 & 36 \\
\hline 6 & 2 & 2 & 1 & 2 & 1 & 1 & 9 & 1 & 0 & 1 & 2 & 156, óbito \\
\hline 7 & 1 & 0 & 0 & 0 & 1 & 2 & 4 & 1 & 0 & 2 & 3 & 6, óbito \\
\hline 8 & 0 & 0 & 0 & 2 & 1 & 1 & 4 & 1 & 0 & 2 & 3 & 8, óbito \\
\hline 9 & 2 & 1 & 2 & 0 & 1 & 2 & 8 & 1 & 0 & 2 & 3 & 60 \\
\hline 10 & 2 & 2 & 2 & 0 & 1 & 2 & 9 & 1 & 0 & 1 & 2 & 36, óbito \\
\hline 11 & 2 & 2 & 2 & 2 & 1 & 1 & 10 & 1 & 0 & 1 & 2 & 48 \\
\hline 12 & 1 & 2 & 0 & 1 & 1 & 0 & 5 & 1 & 0 & 2 & 3 & 2, óbito \\
\hline 13 & 1 & 0 & 0 & 0 & 1 & 2 & 4 & 1 & 4 & 2 & 7 & 36 \\
\hline 14 & 1 & 2 & 2 & 0 & 1 & 0 & 6 & 1 & 4 & 1 & 6 & 12, óbito \\
\hline 15 & 2 & 0 & 0 & 2 & 1 & 0 & 5 & 1 & 0 & 2 & 3 & 6 \\
\hline 16 & 2 & 2 & 0 & 2 & 1 & 2 & 9 & 1 & 0 & 2 & 3 & 24 \\
\hline 17 & 1 & 0 & 0 & 2 & 1 & 2 & 6 & 1 & 0 & 2 & 3 & 36, óbito \\
\hline 18 & 1 & 2 & 2 & 2 & 1 & 2 & 10 & 1 & 0 & 1 & 2 & 3, óbito \\
\hline 19 & 2 & 2 & 1 & 2 & 1 & 2 & 10 & 1 & 0 & 2 & 3 & 8, óbito \\
\hline 20 & 1 & 2 & 2 & 0 & 1 & 1 & 7 & 1 & 0 & 1 & 2 & 20, óbito \\
\hline 21 & 2 & 2 & 0 & 2 & 1 & 1 & 8 & 1 & 0 & 2 & 3 & 4, óbito \\
\hline 22 & 2 & 2 & 2 & 2 & 1 & 1 & 10 & 1 & 0 & 1 & 2 & 26, óbito \\
\hline 23 & 2 & 2 & 0 & 2 & 1 & 2 & 9 & 1 & 0 & 2 & 3 & 5, óbito \\
\hline 24 & 2 & 0 & 0 & 2 & 1 & 1 & 6 & 1 & 0 & 2 & 3 & 7, Óbito \\
\hline 25 & 2 & 2 & 2 & 2 & 1 & 2 & 11 & 1 & 0 & 1 & 2 & 12, óbito \\
\hline 26 & 2 & 2 & 0 & 2 & 1 & 1 & 8 & 1 & 0 & 2 & 3 & 8, óbito \\
\hline 27 & 2 & 2 & 0 & 2 & 1 & 0 & 7 & 1 & 0 & 2 & 3 & 1, óbito \\
\hline 28 & 2 & 2 & 2 & 2 & 1 & 2 & 11 & 1 & 0 & 2 & 3 & 24 \\
\hline 29 & 2 & 0 & 0 & 0 & 1 & 2 & 5 & 1 & 0 & 2 & 3 & 24 \\
\hline 30 & 0 & 1 & 0 & 0 & 1 & 2 & 4 & 1 & 4 & 2 & 7 & 12 \\
\hline
\end{tabular}

Legenda: CG - condição geral; MO - metástases ósseas; MV - metástases vertebrais;

OV - acometimento de órgãos vitais; $1^{\circ}$ - sítio primário; $N$ - lesão neurológica; TOT - total 
TABELA 6 - Resultados da aplicação do escore de Tokuhashi nos pacientes deste estudo

\begin{tabular}{|c|c|c|}
\hline Variáveis & Escore & $\mathrm{N}^{\circ}$ pacientes $(\%)$ \\
\hline \multicolumn{3}{|c|}{ Condição geral } \\
\hline Pobre & 0 & $2(6,7)$ \\
\hline Moderada & 1 & $9(30)$ \\
\hline Boa & 2 & $19(63,3)$ \\
\hline \multicolumn{3}{|c|}{ Metástases ósseas } \\
\hline$>o u=a 3$ & 0 & $10(33,3)$ \\
\hline $1-2$ & 1 & $2(6,7)$ \\
\hline 0 & 2 & $18(60)$ \\
\hline \multicolumn{3}{|c|}{ Metástases vertebrais } \\
\hline$>\mathrm{ou}=\mathrm{a} 3$ & 0 & $17(56,6)$ \\
\hline 2 & 1 & $3(10)$ \\
\hline 1 & 2 & $10(33,3)$ \\
\hline \multicolumn{3}{|c|}{ Metástases de órgãos vitais } \\
\hline Irressecável & 0 & $8(26,7)$ \\
\hline Ressecável & 1 & $1(3,3)$ \\
\hline Ausente & 2 & $21(70)$ \\
\hline \multicolumn{3}{|l|}{ Sítio primário } \\
\hline Mieloma & 1 & $30(100)$ \\
\hline \multicolumn{3}{|c|}{ Alteração neurológica } \\
\hline Completa & 0 & $4(13,3)$ \\
\hline Incompleta & 1 & $12(40)$ \\
\hline Ausente & 2 & $14(46,7)$ \\
\hline
\end{tabular}

ou mais vértebras (nota 0 ), três apresentaram lesão em duas vértebras (nota 1) e dez pacientes $(33,3 \%)$ apresentaram lesão solitária. Para o acometimento de órgãos vitais, oito um paciente $(3,3 \%)$ possuía lesão ressecável (nota 1) e 21 pacientes $(70 \%)$ não apresentaram lesão em órgãos nobres (nota 2).

Quanto ao sítio primário, todos os pacientes receberam a nota 1 , conforme discutido adiante. quatro $(13,3 \%)$ pacientes apresentavam lesão neurológica completa (nota 0 ), 12 tinham lesão incompleta (nota1) e 14 pacientes $(46,7 \%)$ não apresentavam alterações (nota 2) (Tabela 6).

Para o grupo de tratamento não-cirúrgico, a sobrevida prevista é de até três meses. A taxa de sobrevida foi de $57 \%$ aos seis meses, $28 \%$ em 12 meses e nenhum paciente sobreviveu até 36 meses. No grupo intermediário, com sobrevida prevista de até 12 meses, taxa de sobrevida foi $54 \%$ em 12 meses, $31 \%$ em três anos e $24 \%$ em quatro anos. No grupo de tratamento cirúrgico, com sobrevida prevista de mais de 12 meses, a real foi de $60 \%$ em 12 meses, $20 \%$ em 36 meses, $10 \%$ em 48 meses e uma paciente sobreviveu por 156 meses. (Gráfico 1). No escore de Tokuhashi não houve significância estatística para a avaliação da taxa de sobrevida dos pacientes desta amostra $(\mathrm{p}=0,2255)$.

Índice deTomita - Neste escore observamos 27 pacientes com o escore entre 2 e 3 pontos, ou seja, longa sobrevida, três pacientes no grupo intermediário e nenhum no grupo de sobrevida curta. Quanto ao sítio primário todos os pacientes
TABELA 7 - Resultados da aplicação do escore de Tomita nos pacientes deste estudo

\begin{tabular}{lcc}
\hline Fatores prognósticos & Escore & $\mathrm{N}^{\circ}$ pacientes (\%) \\
\hline Sítio primário & 1 & $30(100)$ \\
Metástases viscerais & & \\
$\quad$ Ausente & 0 & $27(90)$ \\
Tratável & 2 & $0(0)$ \\
Intratável & 4 & $3(10)$ \\
Metástases ósseas & & \\
$\quad$ Solitária ou isolada & 1 & $9(30)$ \\
Múltipla & 2 & $21(70)$ \\
\hline
\end{tabular}

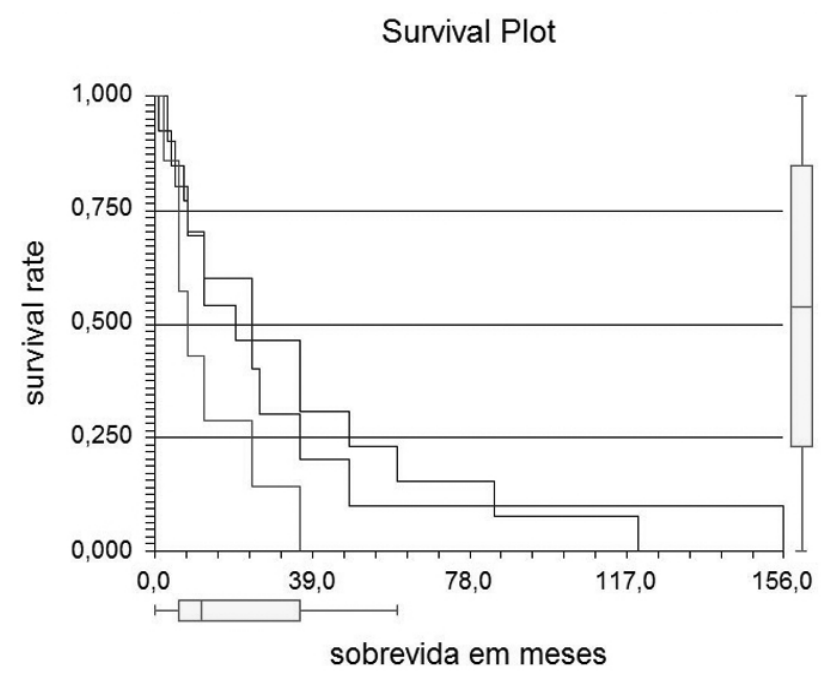

Gráfico 1 - Sobrevida real dos pacientes de acordo com os grupos de Tokuhashi. Linha verde: pior prognóstico; linha azul, prognóstico intermediário; e linha marrom, bom prognóstico

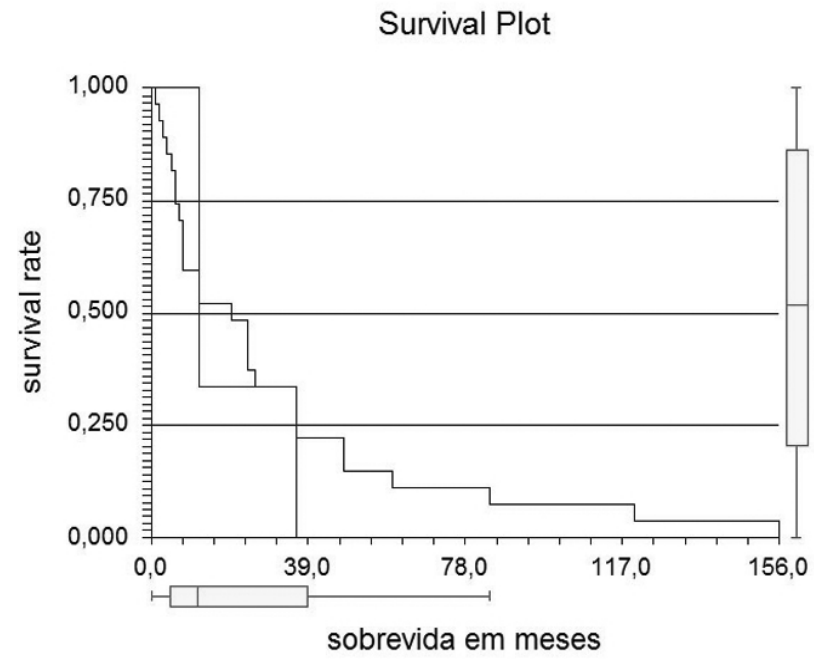

Gráfico 2 - Sobrevida real dos pacientes de acordo com os grupos de Tomita. Linha marrom: pior prognóstico; linha azul, prognóstico intermediário; não houve pacientes com escore de bom prognóstico 
receberam nota 1 , fato que será discutido adiante. Quanto à presença de metástases viscerais, 27 pacientes não apresentavam lesões (nota 0 ), não houve a nota intermediária para lesões tratáveis e os três pacientes restantes apresentavam lesão intratável (nota 4). Quanto às metástases ósseas, nove pacientes (30\%) apresentavam lesão única (nota 1) e 21 pacientes $(70 \%)$ apresentavam lesões múltiplas (nota 2 ) (Tabela 7).

Para o grupo de pior prognóstico, a taxa de sobrevida foi de 33,3\% em doze meses e todos haviam morrido em três anos. Para o grupo intermediário, em 12 meses 52\% estavam vivos, $11 \%$ estavam vivos em cinco anos e apenas um paciente estava vivo após dez anos do diagnóstico da doença. Não houve pacientes no grupo de bom prognóstico (Gráfico 2). No índice de Tomita também não houve significância estatística para a avaliação da taxa de sobrevida dos pacientes desta amostra $(p=0,2255)$.

\section{DISCUSSÃO}

Este estudo foi desenvolvido para avaliar a sobrevida e a aplicabilidade dos escores de Tomita e Tokuhashi em portadores de MM com lesão vertebral, possibilitando dessa forma uma conduta adequada para cada paciente de acordo com a sobrevida predita. Trabalhos prévios avaliaram um foco primário específico, como rim, mama, pulmão, enquanto em outros o foco primário foi variado $o^{4-7,9,15}$. Muitos destes não obtiveram um resultado compatível com os trabalhos originais. Não encontramos na literatura referências sobre a aplicabilidade destes escores no paciente com lesão vertebral e MM. O MM não foi incluído nos estudos originais de Tomita e Tokuhashi. Por isso algumas adaptações foram feitas na tentativa de compatibilizar a doença com os escores de gravidade.

O MM, por ser uma neoplasia primária da medula óssea, difere em alguns aspectos das metástases em geral. $\mathrm{O}$ acometimento em órgãos nobres é infrequente, sendo que apenas o fígado pode estar acometido de uma maneira mais efetiva ( $20 \%$ dos casos), detectado pela hepatomegalia ao exame físico. Os demais órgãos não foram incluídos no estudo pela dificuldade diagnóstica e mínima relação com a sobrevida. As lesões ósseas em geral não são detectadas pela cintilografia, exceção feita em casos de fratura aguda. A identificação das mesmas foi feita através de radiografias simples do corpo inteiro.

A sobrevida média do MM é de 24 a 36 meses, porém em casos sintomáticos e com lesão vertebral ela diminui para seis meses, semelhante à sobrevida da neoplasia primária maligna do fígado. Por isso, a nota atribuída no quesito sítio primário para o MM no escore de Tokuhashi modificado foi 1 e no de Tomita, 1, por analogia. No entanto, esta sobrevida pode variar em razão dos subtipos do MM, dificultando a interpretação desta variável.

No escore de Tomita nenhum paciente pertenceu ao grupo de bom prognóstico devido ao maior valor dado por este autor ao foco primário (até 4 pontos) e a baixa pontuação estipulada ao MM (1 ponto).

O cálculo da sobrevida média não foi realizado porque no final do estudo 12 pacientes estavam vivos. Talvez este resultado pudesse nos levar a uma alteração da nota atribuída ao foco primário, caso a sobrevida média fosse maior do que a encontrada na literatura (seis meses).

A maioria dos trabalhos de avaliação dos escores afirma que o parâmetro "condição geral" deveria receber maior importância na pontuação, tal como recebeu o de "foco primário" na revisão feita por Tokuhashi. Não foi possível concluirmos tal fato em nosso estudo, apesar de admitirmos a relevância do mesmo. Os dois escores não apresentaram significância estatística quanto à aplicabilidade para a amostra que obtivemos. Apesar deste resultado não descartar a possibilidade do uso destes no MM, talvez sejam necessárias novas adaptações a fim de que as sobrevidas real e suposta sejam semelhantes e, portanto, aplicáveis.

Por outro lado, o prognóstico da doença depende de outros fatores não estudados ou inclusos nos escores, como grau de maturidade da neoplasia, estágio da doença no momento do diagnóstico e comprometimento da função renal.

Acreditamos que mais estudos são necessários para a definição de uma estratégia adequada para o tratamento destes pacientes, sem deixar de lado o fato de que a conduta frente a estes casos tão graves é, acima de tudo, individualizada caso a caso. Mesmo com as adaptações feitas para possibilitar a inclusão do MM nestes escores e, provavelmente até por causa destas, nenhum dos escores provou ser estatisticamente significante para a determinação da sobrevida destes pacientes.

\section{CONCLUSÃO}

Neste estudo os escores de Tomita e Tokuhashi não puderam predizer, de forma estatisticamente significante, a sobrevida dos pacientes portadores de MM com lesões na coluna vertebral. Grau de maturidade da neoplasia, subtipo e estágio da doença e principalmente comprometimento da função renal poderiam ser consideradas como variáveis em um escore prognóstico do MM.

\section{REFERÊNCIAS}

1. Harrington KD. Orthopedic surgical management of skeletal complications of malignancy. Cancer. 1997;80(8 Suppl):1614-27.

2. Tokuhashi Y. Letter to the editor. Spine. 2000;25(5):654.
3. Latif T, Hussein MA. Advances in multiple myeloma and spine disease. Clinical lymphoma \& myeloma. 2005;6(3):228-33.
4. Durr HR, Wegener B, Krodel A, Muller PE, Jansson V, Refior HJ. Multiple myeloma: surgery of the spine: retrospective analysis of 27 patients. Spine. 2002 ;27(3):320-4; discussion 5-6. 
5. Roodman GD. Myeloma boné disease: pathogenesis and treatment. Oncology (Williston Park, NY. 2005;19(8):983-4, 6.

6. Ulmar B, Huch K, Naumann U, Catalkaya S, Cakir B, Gerstner S, et al. Evaluation of the Tokuhashi prognosis score and its modifications in 217 patients with vertebral metastases. Eur J Surg Oncol. 2007;33(7):914-9.

7. Tokuhashi Y, Matsuzaki H, Toriyama S, Kawano H, Ohsaka S. Scoring system for the preoperative evaluation of metastatic spine tumor prognosis. Spine. 1990;15(11):1110-3.

8. Hussein MA. Multiple myeloma: most common end-organ damage and management. J Natl Compr Canc Netw. 2007;5(2):170-8.

9. Sundaresan N, Boriani S, Rothman A, Holtzman R. Tumors of the osseous spine. Journal of neuro-oncology. 2004;69(1-3):273-90.
10.Karnofsky DA, Young CW. Comparative aspects of the pharmacology of the antimetabolites. Federation proceedings. 1967;26(4):1139-45.

11. Tokuhashi Y, Matsuzaki H, Oda H, Oshima M, Ryu J. A revised scoring system for preoperative evaluation of metastatic spine tumor prognosis. Spine. 2005;30(19):2186-91.

12.Tomita K, Kawahara N, Kobayashi T, Yoshida A, Murakami H, Akamaru T. Surgical strategy for spinal metastases. Spine. 2001;26(3):298-306.

13.Ulmar B, Naumann U, Catalkaya S, Muche R, Cakir B, Schmidt R, et al. Prognosis scores of Tokuhashi and Tomita for patients with spinal metastases of renal cancer. Annals Surg Oncol. 2007;14(2):998-1004.

14.Enkaoua EA, Doursounian L, Chatellier G, Mabesoone F, Aimard T, Saillant G. Vertebral metastases: a critical appreciation of the preoperative prognostic tokuhashi score in a series of 71 cases. Spine. 1997;22(19):2293-8.
15.Ogihara S, Seichi A, Hozumi T, Oka H, Ieki R, Nakamura K, et al. Prognostic factors for patients with spinal metastases from lung cancer. Spine. 2006;31(14):1585-90.

16.Ulmar B, Richter M, Cakir B, Muche R, Puhl W, Huch K. The Tokuhashi score: significant predictive value for the life expectancy of patients with breast cancer with spinal metastases. Spine. 2005;30(19):2222-6.

17.Flemming DJ, Murphey MD, Carmichael BB, Bernard SA. Primary tumors of the spine. Seminars in musculoskeletal radiology. 2000;4(3):299-320.

\section{Correspondência}

Marcos Vaz de Lima

Rua Dr. Cesáro Mota Junior 112

São Paulo (SP), Brasil.

CEP: 01277-900

Tel.: + 551121761565

E-mail: coluna@santacasasp.org.br; robertmeves@hotmail.com;

vaz_de_lima@hotmail.com 